\title{
Carnets
}

Revue électronique d'études françaises de l'APEF

Première Série - $1 \mid 2009$

La mer... dans tous ses états

\section{Fictions sur mer. Place de la mer dans l'écriture minimaliste de Francis Dannemark}

José Domingues de Almeida

\section{(2) OpenEdition}

Journals

Édition électronique

URL : http://journals.openedition.org/carnets/2724

DOI : $10.4000 /$ carnets. 2724

ISSN : 1646-7698

Éditeur

APEF

Édition imprimée

Pagination : 7-16

\section{Référence électronique}

José Domingues de Almeida, «Fictions sur mer. Place de la mer dans l'écriture minimaliste de Francis

Dannemark », Carnets [En ligne], Première Série - 1 | 2009, mis en ligne le 13 juin 2018, consulté le 02 mai 2019. URL : http://journals.openedition.org/carnets/2724; DOI : 10.4000/carnets.2724

\section{(c) (1) (8)}

Carnets est mis à disposition selon les termes de la licence Creative Commons - Atribution - Pas d'utilisation commerciale 4.0 International. 


\section{FICTIONS-SUR-MER}

\section{Place de la mer dans l'écriture minimaliste de Francis Dannemark}

José DOMINGUES DE ALMEIDA

Universidade do Porto

jalmeida@letras.up.pt

\section{Résumé}

La mer et la plage jouent un rôle très particulier dans le décor et la fiction minimalistes de l'œuvre fictionnelle de l'écrivain belge francophone contemporain Francis Dannemark en tant que lieu d'indéfinition spatiale et de fuite fantasmatique. Notre approche critique tâchera de l'illustrer dans plusieurs romans unanimement considérés "minimalistes" et "postmodernes" par la critique. L'auteur tâche d'illustrer la technique narrative à l'œuvre dans les romans de Dannemark, ainsi que la vision spécifique et ambiguë de la Belgique qui s'en dégage par le biais du motif de la mer.

\section{Abstract}

The sea and the beach play a very specific role in the minimalist scenes and fiction of the contemporary Belgian francophone writer Francis Dannemark. They represent both space undefinition and fantasmatic escape. Our critical approach will try to illustrate that in several Dannemark's novels unanimously considered as "minimalist" and "postmodern" by criticism. The author tries to illustrate the narrative technique present in these novels as well as the specific and ambiguous vision of Belgium emerging from them through the theme of the sea.

Mots-clés: Dannemark, Postmodernité, Minimalisme, Mer, Belge

Keywords: Dannemark, Postmodernity, Minimalism, Sea, Belgian 
Ayant commencé à publier des romans dans le tournant des années quatre-vingt, Francis Dannemark fait partie d'une nouvelle génération d'écrivains belges francophones émancipée du poids des questions identitaires liées au débat et au concept de la belgitude, mais aussi "français" pris dans un nouveau rapport à la modernité romanesque et au jeu narratif hérité du nouveau roman et des avant-gardes textuelles des années septante.

Né en 1955 dans le Hainaut belge, mais Bruxellois depuis toujours, Dannemark commence à publier plusieurs recueils de poésie vers la fin des années septante, avant de faire alterner poésie et prose romanesque à partir des années quatre-vingt avec des titres comme Le voyage à plus d'un titre (1981), La nuit est la dernière image (1982), Mémoires d'un ange maladroit (1984), Choses qu'on dit la nuit entre deux villes (1991) ou Qu'il pleuve (1998).

D'une part, Francis Dannemark s'inscrit dans cette génération d'écrivains belges pour qui les interrogations et les combats identitaires n'ont plus beaucoup de sens d'autant plus que la belgité, sentiment positif et décomplexé de l'appartenance à l'ici belge, surgi après la belgitude, n'est pas sans lien avec l'émergence postmoderne d'un certain consensus scriptural à partir des années quatre-vingt, lequel parodie ou minimise le travail moderne de l'écriture, et le contenu revendicatif lié aux démarches textuelles.

À ce propos, Jacques Dubois avait tôt manifesté sa crainte de voir peu à peu le "grand consensus scriptural" dissiper la littérature belge de langue française dans une espèce de littérature moyenne, et effacer ou taire la voix belge, et les spécificités francophones en général, dans l'innocuité postmoderne (1985: 37).

Christian Prigent avait, à cet égard, dénoncé l'infléchissement du moderne qui fait en sorte que, dans un consensus inoffensif et largement pratiqué, l'écriture tende à devenir "lisible', amical[e], apaisé[e]" (1991: 121). II semble, vu l'essor de ce type d'écriture en Belgique depuis les années quatre-vingt, que cette appréhension ne se soit pas tout à fait avérée, même si la tendance minimaliste le laisse penser.

Dans ce double contexte, le débat identitaire se voit amputé des conditions de se tenir. D'ailleurs, l'abandon d'un discours de type identitaire et/ou revendicatif par les acteurs littéraires devient le dénominateur commun des nouvelles générations d'écrivains (Amélie Nothomb, Eugène Savitzkaya, Jean-Philippe Toussaint, Francis Dannemark, etc.).

Autrement dit, l'écriture dannemarkienne acte ce phénomène général de l'effacement des appartenances identitaires nationales dans les espaces francophones à la double faveur du consensus postmoderne de l'écriture, mais aussi de l'infléchissement de la puissance symbolique française après le verbe gaullien, ce qui a eu pour effet d'atténuer le magnétisme des instances parisiennes de reconnaissance (du centre) sur les périphéries francophones, et la marge littéraire belge au premier chef.

D'autre part, comme nous le signalions plus haut, l'écriture de Dannemark ressortit à 
un cadre nouveau de l'écriture romanesque dans la foulée de l'esthétique du nouveau roman parisien et de ses conventions et prémisses, mais déjà en quête d'autre chose sur le plan narratif proprement dit.

Déjà en 1991, le premier numéro de la revue liégeoise Écritures proposait un article tâtonnant de Laurent Demoulin intitulé "Pour un roman sans manifeste" où le retour du récit est perçu sous trois formes distinctes, liées à l'incontournable renouveau de Minuit et à son onde de choc sur d'autres jeunes écrivains arrivés sur la scène littéraire (1991: 8-21).

La première tendance a trait à ce que Demoulin désigne par "l'écriture du piétinement" (ibid:: 16): piétinement du récit autour d'un héros hésitant et irrésolu. Une description que d'aucuns feront coïncider avec l'écriture épurée, dite minimaliste ou "blanche", et dont rend bien compte La salle de bain du Belge Jean-Philippe Toussaint, ainsi que celle à l'œuvre dans certains romans de Gailly, Costa ou Oster.

La seconde tendance dégagée par Demoulin se réfère au travail scriptural de détournement et de surcodage du récit par le biais d'un clin d'œil intertextuel. Cette pratique véritablement postmoderne (dans le sens immanent du terme, celui du seul contexte littéraire) correspond bien aux romans d'Echenoz ou ceux de Deville dont l'intrigue s'apparente à la fausse anecdote ou se termine en queue de poisson.

Finalement, Demoulin souligne une tendance plus ou moins autobiographique en harmonie avec le retour annoncé du sujet, mais consciente, après la psychanalyse, des limites de la représentation du moi. Des écrivains tels que Guibert, Rouaud ou encore le Belge Blasband pratiqueraient cette autobiographie détachée où évolue un "je" d'une neutralité ou d'une impassibilité inédites.

En fait, Laurent Demoulin devait préciser plus tard les taxinomies de ces contemporanéités littéraires qu'il désigne plus clairement par écriture postmoderne et minimaliste (1997: 7-17) et dont il décrira les caractéristiques majeures et floues: auteurs nés autour de 1955, traitement parodique infligé à l'anecdote, impassibilité angoissée du héros, autoréflexivité consciente des codes (réalistes et modernes), phrasé correct du style, dépouillement des moyens narratifs allié à l'immobilité de l'action et des personnages.

Les romans parus chez les Éditions de Minuit des Belges Jean-Philippe Toussaint (La salle de bain) et d'Eugène Savitzkaya (Un jeune homme trop gros), ou du Français Jean Echenoz (Le méridien de Greenwich) avaient ouvert une brèche esthétique romanesque dans laquelle d'autres viendront vite s'engouffrer. Demoulin rappelle, à cet égard, que "la littérature belge, qui a payé un large écot à la modernité [...] est également aux premiers rangs de la postmodernité [...]" (ibid.: 11). II ne cite bizarrement pas Dannemark, mais tout son raisonnement porte à accréditer l'intuition d'une accointance minimaliste de son écriture romanesque.

De son côté, Jacques-Pierre Amette (1995) a dégagé la taxinomie critique de ce 
dernier sursaut de la modernité littéraire: évitement du modèle (post)balzacien, quête scripturale d'"autre chose", impassibilité de l'écrivain et du personnage, recours au rythme et à la technique du cadrage cinématographique, minimalisme généralisé et affiché de l'économie textuelle et narrative.

Michel Crépu (2001) brossera, pour sa part, un judicieux portrait-robot de l'écrivain minimaliste français, lié le plus souvent aux Éditions de Minuit: héritage beckettien, épurement du réel, la brièveté narrative se traduisant par des histoires courtes et condensées, des "courts-métrages" en somme.

Ces caractéristiques minimalistes que Fieke Schoots (1994) a abondamment inventoriées et illustrées dans la fiction narrative des auteurs de Minuit de ces dernières années se retrouvent toutes en puissance dans les textes narratifs dannemarkiens: la brièveté de l'agencement narratif (les romans dannemarkiens ne dépasseront pas les cent cinquante pages), le dépouillement des moyens narratifs, le phrasé hypercorrect, l'humour subtil sous la parodie et l'impassibilité du narrateur-personnage, mais aussi le complexe rapport à l'espace et aux lieux traversés dans le roman dit minimaliste ou dans l'écriture postmoderne française.

C'est à l'aune de cette dernière composante narrative minimaliste que nous tâcherons d'approcher la place tout à fait particulière occupée par l'isotopie maritime dans ces textes romanesques. Notre lecture se portera sur les romans Le voyage à plus d'un titre (1981), La nuit est la dernière image (1982 et réédité en 2007), Mémoires d'un ange maladroit (1984 et réédité en 1993), Choses qu'on dit la nuit entre deux villes (1991).

Cette caractéristique a partie liée avec la conception même du héros minimaliste, impassible et humoristique en général, et avec sa construction spécifique chez Dannemark. Car c'est aussi du côté du personnage et de sa focalisation que se joue le minimalisme comme l'a si bien illustré Fieke Schoots (1994).

Déprimé, perdu, pris dans un jeu de hasard: "Ce héros dannemarkien mène une existence sans conclusions. Aussi ces textes ont-ils donné à quelques lecteurs pressés le sentiment du désinvolte, sinon du superficiel" (Laroche, 1991: 8).

L'absence de toute anthropologie profonde et psychologisante, héritage du nouveau roman, laisse libre cours à ce que "les êtres qui les [ces textes] peuplent paraissent sans poids, sans gravité [...]" (ibid.). De ce fait, le héros dannemarkien s'abandonne plutôt aux détails et aux nuances: couleurs, musicalités ou l'attention portée aux points de bascule chronologiques et météorologiques: "La route tourne et tourne encore. Parfois c'est la mer qui apparaît, parfois des rideaux d'arbres, quelques maisons, - et la voiture roule lentement, régulièrement, sans autre but apparemment que suivre la musique que Wolf a mise à la place des airs de jazz" (Dannemark, 1991: 86 et ss.).

Ce souci de l'alternance climatique fonctionne comme une véritable marque voulue 
de l'entre-deux des saisons et des heures du jour, "moments de transition, marqués par une sorte de précarité qui rend d'autant plus précieux" (Laroche, 1991: 18); "Le printemps qui se termine est très lent" (Dannemark, 2007: 54); "À la fin de l'hiver, c'est la mer qui est en vacances" (Dannemark,1991: 15).

Ce qui permet d'introduire la pertinence narrative d'un lieu particulier, la mer, dont l'attachement au cadre national n'est pas toujours assuré; une indéfinition qui concourt à l'édification d'un mythe plus qu'à celle d'un simple décor: "J'avais presque cessé de prêter attention à la mer quand une vague plus haute vint brutalement m'éclabousser. Avec elle croula d'un seul coup tout ce qui restait du vaste château de sable" (Dannemark,1993: 16); "D'ici, la mer n'est pas la mer aujourd'hui, mais un tableau qui représente, immobile dans l'encadrement de la fenêtre. J'aimerais que vous soyez ici avec moi pour le regarder" (Dannemark, 2007: 69).

En fait, s'agissant de la fiction narrative de Francis Dannemark, il y a tout lieu de parler de "fictions sur mer" tant le décor maritime constitue un élément central de la construction du récit et s'assume, au-delà du simple cadre, en personnage latent, tacite, présent, ne serait-ce qu'en creux.

La biobibliographie de l'auteur fait apparaître un attachement particulier à cette région du littoral belge, en Flandre, mais intimement associé à l'image d'une Belgique mythique et unitaire d'antan, royaume d'avant la scission fédérale des successives réformes de l'État belge.

Aller sur la "côte", notamment à Ostende, zone bilingue, voire francophone pour les élites flamandes, renvoie à un album photographique national désormais périmé, ou en tous cas fortement mis en cause par la terrible dislocation de l'État. Un coup d'œil sur les documents photographiques illustrant la biographie de l'auteur pour l'édition Labor de Mémoires d'un ange maladroit suscite l'évocation de souvenirs personnels de séjours familiaux sur la côte belge dans les années soixante et septante, mais qui ne sont pas sans éveiller un cadre national révolu, marqué par l'entretien d'une cohérence et d'une cohésion territoriales et idiosyncrasiques dans les faits et dans les têtes, et que l'on a souvent désigné par "la Belgique de papa", parfois regrettée.

Ce contexte passé, tant national que personnel, permet d'interroger l'inscription purement indicielle de l'ici dans ces récits minimalistes, surtout dans la prégnance de la mer. Ce motif récurrent s'avère moins un repère qu'un point de fuite. II cristallise l'indéfinition territoriale comme corollaire de l'absence d'une véritable anthropologie dans le roman minimaliste, et en devient dès lors un élément constitutivement narratif.

Rappelons que le cadre maritime se trouve présent dans tous les romans dannemarkiens. II s'agit donc d'une véritable constante narrative et fantasmatique, liant écriture romanesque et autobiographie; un cadre personnel contaminant irrépressiblement le 
décor narratif.

Dans Le voyage à plus d'un titre (1981), premier roman de Francis Dannemark, un photographe free lance et globetrotteur prisé, notamment suite à une photo dans le cadre de guerre libanais, délaisse soudain et inexplicablement sa carrière. Au volant de sa voiture, il se lance dans le réseau labyrinthique des autoroutes, que l'on devine belges par certaines caractéristiques, notamment par le relief plat et rural où "l'autoroute tranche en ligne droite une étendue de champs..." (ibid.: 57).

Ce périple maniaco-dépressif, qui n'est pas sans rappeler le héros de La salle de bain (1985) du Belge Jean-Philippe Toussaint, qui ne voulait pas quitter sa salle de bain, est ponctué par plusieurs appels téléphoniques d'amis inquiets. Cette fascination suicidaire pour les "non-lieux" anthropologiques (Augé, 1992: 103), notamment l'autoroute, conduit le héros vers un littoral imprécis, mais devinable: "Pas d'horizon. II faudrait tourner tout à fait la tête, chercher des falaises [horizon anglais], points blancs minuscules à des kilomètres de là" (Dannemark, 1981: 27); destination avortée de ce voyage immobile et scriptural: "Un vertige lui fait fermer les yeux. II se voit à la pointe d'un brise-lames, face à la mer qui remonte. II faut terminer la conversation, dire qu'on se verra bientôt, oui, bientôt" (ibid.: 99).

Dans ce récit, où se révèlent la plupart des caractéristiques du personnage minimaliste que nous résumions plus haut par le terme "impassibilité": humour inquiet, incapacité d'établir des rapports durables, phobies et dépressions diverses, l'hypothétique but du littoral apparaît comme une possible délivrance, un salut, qui sera reporté à une sortie d'autoroute.

La mer s'oppose ainsi, par son immensité et son ouverture, à l'étroitesse et à I'homogénéité étouffante et hermétique des circuits autoroutiers. Elle constitue l'issue rêvée du labyrinthe spatial et ontologique. Une échappatoire et un défoulement par rapport à l'exiguïté territoriale condensée, voire métaphorisée par le système dédalique du réseau autoroutier. Sa vue est l'occasion d'un espoir, d'un répit et d'un "vertige".

Dans La nuit est la dernière image (1982 \& 2007), David et Anne se retrouvent lors d'un périple dans un désert africain. Des troubles politiques inidentifiables repoussent leur retour au pays (mais quel pays?). Pendant ce temps, il faut se retrancher à l'hôtel, lire et écrire, tuer le temps avec des dialogues qui ne mènent à aucune relation durable, et contempler un espace désertique changeant.

L'absence de mer dans ce cadre désertique n'en suggère pas moins le désir de sa contemplation, de son ouverture. À la faveur d'un entre-deux météorologique, la tombée du soir, David se met à imaginer, par extrapolation, l'effet de cette apparition, et de sa fuite personnelle, jusqu'à la mer qu'il sait lointaine, mais qu'il appelle de ses fantasmes intimes: "Au bout de l'allée, le palmier a disparu dans l'ombre. La nuit est tombée sur le jardin, sur la terrasse. Sur le désert et sur ses routes incertaines [...]. II finirait par atteindre les premières 
dunes. Il tomberait, il mourrait, le visage enfoncé dans le sable" (ibid.: 36).

Le début du récit met, d'emblée, le couple dans un état d'extranéité, de dépaysement mesurable par la comparaison des paysages maritimes; celui de ce pays où il se trouve à celui d'où il vient, tous deux indéfinissables: “Une mer de sable, mais qui n'a rien à voir avec la mer, celle qu'il imagine, qu'il revoit, grise, bordée de plages blanches où les pas des rares promeneurs de l'hiver dessinent de longues et dérisoires figures" (ibid:: 7).

La mer ne se voit pas, surtout quand on n'est pas chez soi. Elle se laisse deviner et désirer: "Pour le reste, la nuit à perte de vue, jusqu'à la mer qu'on ne voit pas" (ibid:: 47). Mais le retour en zone, le retour au pays indéterminé du récit suppose les retrouvailles d'une mer amadouée, aux teintes habituelles, nordiques et septentrionales: "Le ciel bleu ardoise, la mer gris cendre. Le vent souffle lentement vers les dunes. La plage, à marée basse, s'étend loin. II marche sur la frontière du sable sec et de la zone d'où la mer s'est retirée, dessinant des vagues sur le sable mouillé" (ibid:: 66).

De retour de ce chez eux innommé, c'est un décor de côte belge qui domine le récit et concourt à l'édification minimaliste du récit: "Ils y vont en empruntant la digue. Sur la plage, quelques promeneurs. Plus loin, au bord de l'eau, court un homme en vêtement de sport" (ibid.: 75); "La mer est à peine visible, une masse sombre tachée d'écume" (ibid.: 78), de telle sorte que le dénouement de ce roman sans intrigue se termine sur un décor maritime belge non déclaré: "Par la porte vitrée, Anne regarde la digue, la mer intacte, sans bruit, elle referme le livre" (ibid:: 86).

Dans Mémoires d'un ange maladroit (1984 \& 1993), un vieillard, Hermann, au passé énigmatique, invite le narrateur, Harry, et Cathy à organiser ses archives personnelles faites de plusieurs histoires éparses dans des films, des lettres, des notes, des billets, des agendas, des carnets et autres matériaux biographiques et... scripturaux disparates.

Ce travail devient le prétexte de rapports éphémères entre les deux archivistes en herbe, et est le prétexte à quelques dialogues brefs, minimaux. Mais c'est le décor maritime de cette intrigue au minimum qu'il convient de retenir ici et qui renvoie à une "tripartition des lieux" (Bertrand, 1993: 182): la ville, la villa d'Hermann et la mer. En effet, le récit commence, juste après l'incipit, et se termine sur une vision de cette mer du Nord si chère à l'écrivain: "J'avais presque cessé de prêter attention à la mer quand une vague plus haute vint brutalement m'éclabousser" (Dannemark, 1993: 16); "Juste au-dessus, sur le bleu de la mer, je notai au crayon le nom d'Anna" (ibid:: 160).

Ce cadre nordique belge, inavoué, mais facilement perceptible par sa prégnance autobiographique: "Je prends la voiture et j'emprunte la route qui longe la côte, dans ce brouillard qui me plaît parce qu'il n'efface pas complètement le décor, mais qu'il lui donne un caractère à la fois impalpable et indestructible" (ibid.: 69), s'avère très vite l'ambiance choisie pour l'élaboration fictionnelle et hypothétique de ces bribes de biographie ou d'archives 
d'Hermann. Un château de sable est sans cesse construit et détruit sur la plage. Son côté éphémère et gratuit renvoie immanquablement au statut provisoire et discontinu de l'écriture romanesque, surtout dans sa version minimaliste et postmoderne. Tout semble voué à l'échec et à l'essai arbitraire: "J'écris pour rien les mémoires de personne" (ibid.: 80).

Le temps de ce récit, qui procure trois dénouements possibles au lecteur, et qui se présente de façon triangulaire comme nous le faisions remarquer, fait apparaître la mer, la plage et le rivage en général comme lieux privilégiés d'une sortie et d'une fuite réflexive et sentimentale par rapport à la présence hautaine et close du château où logent les personnages.

Celui-ci connaît d'ailleurs son corollaire symbolique et fantasmatique dans le château de sable qui se construit et est démoli sur la plage: "Un long moment, j'ai observé de loin un homme en train de bâtir un extraordinaire château de sable. II devait être là depuis l'aube" (ibid:: 30); ce qui assigne à l'“architecte" qui le projette sans cesse, le statut de double ou d'alter ego du narrateur-archiviste tant les deux tâches se ressemblent et se rejoignent en précarité face à la mer: "Il était là, sur la plage, agenouillé, creusant de petites fenêtres à l'aide d'une mince planchette en bois, du genre de ces abaisse-langue qu'emploient les médecins" (ibid.: 94); "Faire quelque chose avec ces notes? J'en prends de moins en moins. Terrassier, je comble des trous, tant bien que mal. J'improvise et j'emprunte; j'emprunte et j'invente" (ibid.: 114).

De ce fait, la mer est omniprésente et sert non pas seulement de cadre, mais de repère existentiel au narrateur: "Lentement, je me décomposais, je disparaissais, le matin viendrait et tout aurait disparu, comme un mouchoir en papier emporté par l'eau jusqu'à l'eau des égouts et des égouts jusqu'à l'eau salée de la mer" (ibid:: 51). Elle s'assume comme destin inéluctable de toute action humaine; de toute existence tout comme elle s'avère le réceptacle de toutes choses.

Enfin, dans Choses qu'on dit la nuit entre deux villes (1991 \& 1996), le lecteur retrouve les mêmes procédés de triangulation narrative (un couple se connaît lors des préparatifs du mariage d'amis communs) et spatiale (les routes, la villa et la plage): "Quelle folie, dit Lena en portant son regard de la maison vers la mer" (Dannemark, 1991: 18). En fait Wolf et Lena font connaissance autour d'un mariage sans cesse reporté et finalement annulé, et qui procure l'occasion d'échanger ces "mots" au hasard d'une randonnée en voiture ou d'une promenade sur la plage.

Ces deux personnages se retrouvent ainsi dans un décor de fin de saison, sur la côte (belge?) désertée par les vacanciers à cette époque de l'année. La fiction sur mer devient une conversation au bord de la mer, mais sans prétention sentimentale. Tout se joue, comme dans le minimalisme romanesque français en général, dans l'effleurement, l'allusion et l'impassibilité. 
Mais à nouveau, c'est la mer qui offre un cadre et s'impose comme une présence indéniable dans ce récit: "Le fond musical, c'est la mer" (ibid.: 37). Comme à l'accoutumée chez cet écrivain belge, les mutations saisonnières et le poids narratif et symbolique de la mer vont de pair: "À la fin de l'hiver, c'est la mer qui est en vacances" (ibid.: 15). C'est elle qui rythme les mouvements et les répits des villas louées en vue des noces et qui restent désespérément vides: "Tout le monde est parti et le calme silence qui vient de la mer met en relief les musiques dont Wolf et Lena remplissent la villa" (ibid.: 45).

Encore une fois, la mer et son réseau isotopique viennent colmater les nombreuses brèches diégétiques et recadrer le récit: “Le mariage est annulé', dit-il en s'installant à nouveau dans la voiture. Lena veut savoir, il dit qu'il va lui raconter en roulant, on va voir le port, on se promènera un peu, je partirai ce soir. Et quelques minutes plus tard, sur la route qui longe la côte, il raconte à Lena..." (ibid.: 83).

C'est, dès lors, le récit qui, d'une certaine façon, finit par longer la mer: "La route tourne et tourne encore. Parfois c'est la mer qui apparaît..." (ibid.: 86); "Wolf ouvre la vitre, le bruit de la mer entre dans la voiture avec des bouffées d'air frais" (ibid.: 96).

C'est dire combien, chez Francis Dannemark, la mer dépasse de loin le simple statut de décor narratif pour atteindre à autre chose qui est de l'ordre de l'inscription du vécu et, dès lors, de celui d'un lieu, d'un ici national que la nouvelle mouvance d'écrivains belges ne ressent plus le besoin de nommer, mais qui n'en demeure pas moins un puissant symptôme et surtout, dans ce cas spécifique, un incontournable repère, à plus d'un titre: "Je n'ai rien écrit sur la vitre embuée ce jour-là, et j'ai su que j'étais heureux, et heureux d'aimer, et la vie était là, dans l'amour, avant d'un jour rejoindre le souffle splendide et définitif de la mer" (ibid:: 108).

Ce faisant, ces textes pointent un dépassement du cadre d'inscription ou de lecture nationale de la littérature, notamment en Belgique, où le débat identitaire bien daté de la belgitude avait voulu placer le fait littéraire, et illustrent un souci autre de l'écriture contemporaine. 


\section{Bibliographie}

AMETTE, Jacques-Pierre (1995). “Une certaine tendance du roman français". In: Le Point, 11/03/1995.

AUGE, Marc (1994). Não-lugares. Introdução a uma antropologia da sobremodernidade. Lisboa: Bertrand Editora.

CREPU, Michel (2001). "Le roman français est-il mort?". In: L'Express, 29/03/2001.

DANNEMARK, Francis (1981). Le voyage a plus d'un titre. Paris: Robert Laffont.

DANNEMARK, Francis (1982, réédité en 2007). La nuit est la dernière image. Bruxelles: Le Castor Astral.

DANNEMARK, Francis (1984, réédité en 1991). Choses qu'on dit la nuit entre deux villes. Paris: Robert Laffont.

DANNEMARK, Francis (1993). Mémoires d'un ange maladroit (suivi d'une lecture de P. Bertrand). Bruxelles: Labor.

Demoulin, Laurent (1997). "Génération innommable”. In: Textyles, no 14, Lettres du Jour (II).

DuBOIS, Jacques (1985). "Jeu de forces et contradictions dans le champ littéraire de la Belgique contemporaine". In: Trajectoires: Littérature et Institutions au Québec et en Belgique francophone. Bruxelles: Edition Lise Gauvin et Jean-Marie Klinkenberg.

DuBoIS, Jacques et al. (1997). "Écrire en Belgique. Une autonomie à la carte". In: La Revue Nouvelle, ํㅡ 3, mars 1997.

LAROCHE, Daniel (1991). La mise à distance. Dits et non-dits dans l'œuvre de Francis Dannemark. Bruxelles: Promotion des Lettres.

PRIGENT, Christian (1991). Ceux qui merdRent. Paris: P.O.L.

ScHOOTS, Fieke (1994). "L'écriture 'minimaliste'”. In: Jeunes auteurs de Minuit. Amsterdam et Atlanta: Rodopi.

SchoOTs, Fieke (1997). "Passer en douce à la douane". L'écriture minimaliste de Minuit. Amsterdam et Atlanta: Rodopi. 